\title{
The extent of 3-min oscillations in regions other than sunspot plumes
}

\author{
C.-H. Lin $^{1}$, D. Banerjee ${ }^{2}$, J. G. Doyle ${ }^{1}$, and E. O’Shea ${ }^{1}$ \\ 1 Armagh Observatory, College Hill, Armagh BT61 9DG, N. Ireland \\ e-mail: chl@arm.ac.uk \\ ${ }^{2}$ Indian Institute of Astrophysics, Koramangala, Bangalore 560034, India
}

Received 30 May 2005 / Accepted 12 August 2005

\begin{abstract}
The active region, AR0554, was observed with NIS/CDS on board SoHO to examine the extent and range of oscillations from a range of features. Among all the NIS spectral lines analysed, significant oscillations were found in Si XII $520 \AA$, Mg X $625 \AA$, O v $629 \AA$, and He I $522 \AA$. The periods of the strongest oscillations in these lines were $\approx 10-20 \mathrm{~min}$. After the dominant $10-20$ min oscillations were filtered out from these lines, only O V $629 \AA$ showed significant (i.e. above the 95\% significance level) shorter-period oscillations. Specifically, we found that weak but significant 3-min oscillations are not confined to the umbra/plume but can be seen in many bright locations. The duration of these non-umbral 3-min oscillations is mostly $\approx 20 \mathrm{~min}$. In contrast, the 3-min oscillations within the umbra are strong and stable for longer than $50 \mathrm{~min}$. The duration could be related to the size of the oscillating source region, rather than the lifetime of the oscillation. To find the possible sources of these 3-min oscillations outside of the umbra, we compared the oscillations of single pixels in different regions. The results of our comparison indicate a possible connection between the magnetic fields and the oscillations. Therefore, we suggest that 3-min oscillations may exist in many magnetic structures, but are often too weak to be seen in an unfiltered signal.
\end{abstract}

Key words. Sun: corona - Sun: oscillations

\section{Introduction}

The detection of waves in the solar atmosphere has been of great interest to both solar and plasma physicists. Observations of oscillatory phenomena in the solar atmosphere have increased dramatically in the past few years. Detailed observational studies, especially with the Solar and Heliospheric Observatory (SoHO) and the Transition Region And Coronal Explorer (TRACE) have provided a strong stimulus to theoretical developments. A new field of study, namely, coronal seismology has emerged, which allows the determination of coronal parameters by using information about the properties of the waves that the medium supports. This field is rapidly developing and providing insights into the processes at work in the corona.

Mode frequencies and damping rates are two main observable properties of the waves, and, hence, are important in linking theoretical models with observations. Most of the observations to date have suggested that 3-min oscillations are associated with sunspots. De Moortel et al. (2002) reported the detection of 3-min oscillations in the coronal loops above sunspots and 5-min oscillations in other coronal loops. The observational results in Banerjee et al. (2002) indicate that 3-min oscillations are localized within the umbra. Maltby et al. (1999) found 3-min oscillations in coronal loops that are rooted in the umbra. By examining TRACE and CDS data, Brynildsen et al. (2002) found that the 3-min oscillations of transition region lines are present in the whole umbral region but the 3-min oscillations of coronal lines tend to localize in small regions that coincide with the endpoints of coronal loops. In their study, the 3-min oscillations were not observed with CDS at coronal temperatures within the spots, but were seen in the TRACE $171 \AA$ band. In a different joint observing campaign with CDS and TRACE, O'Shea et al. (2002) reported the detection of 3-min oscillations over a larger sunspot as seen in Fe XVI $335 \AA$, a high temperature coronal line in CDS. This could indicate that the detection of oscillations crucially depends on the extent of the oscillating region.

Most of the oscillations reported in previous papers are the dominant oscillations in a signal. However, a signal can be a composition of many oscillation modes. The modes with smaller amplitudes can also provide important information, and, therefore, should not be overlooked. In addition, the energy distribution over different modes can provide insight into the structure of the plasma. In this study, our NIS/CDS (Normal Incidence Spectrometers) observation covered different features in an active region that contains a 

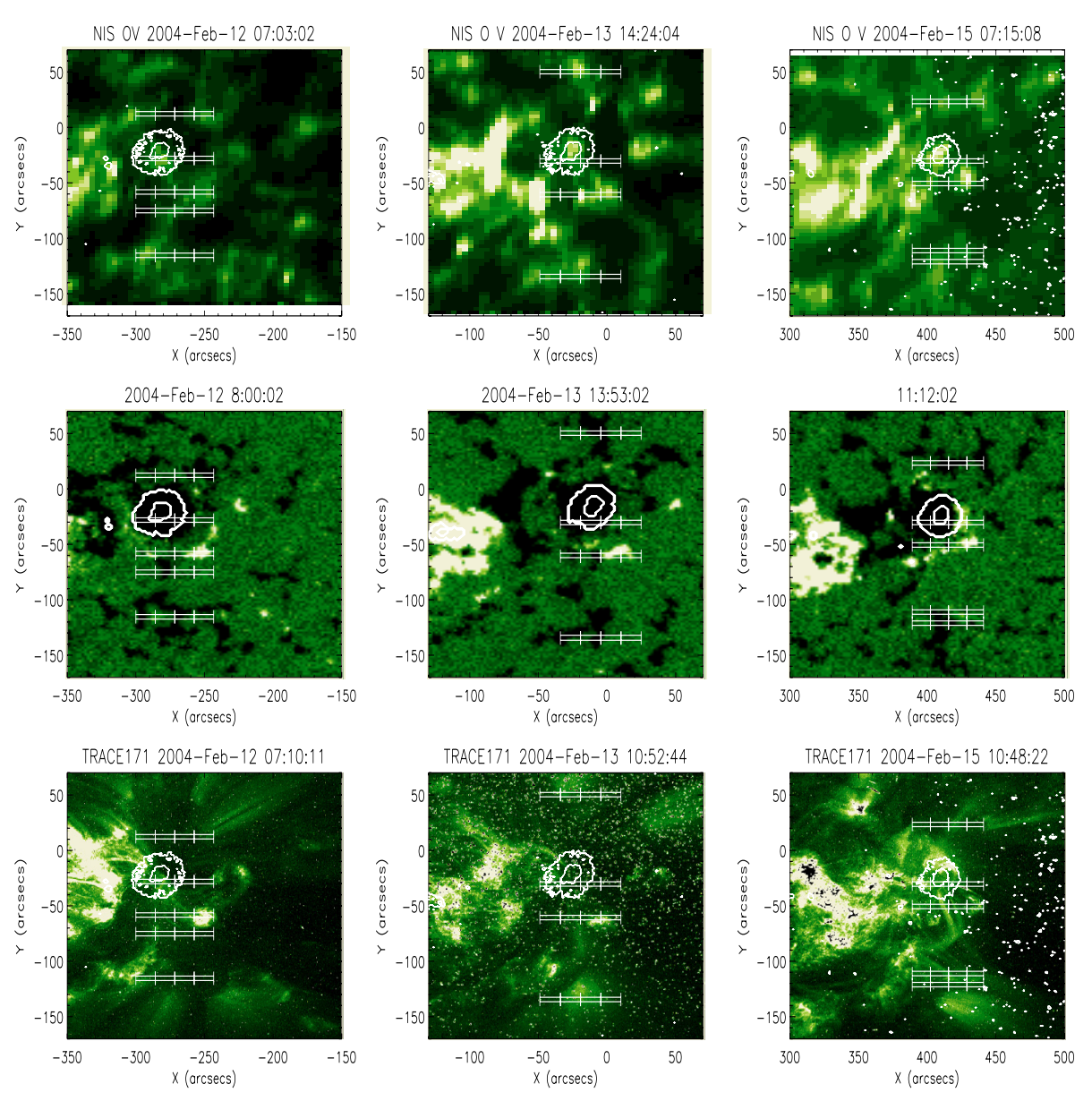

Fig. 1. The maps (from top to bottom rows) of NIS O v 629, MDI magnetograms, and the TRACE $171 \AA$ channel (North is up, East to the left). Each map is selected to represent the features in one of the following days: Feb. 12th, 13th, and 15 th. The actual observation time of each map is indicated above the corresponding map. The contours are the outlines of the umbra and penumbra. We mark the locations of the selected pixels with long, thin rectangular boxes. The vertical length of a box is the size of a pixel. There are four divisions in each box, which correspond to the FOV of the four time series. sunspot plume. Our analysis shows that the transition region line O V $629 \AA$ contains many different modes while the coronal lines, Si XII $520.8 \AA$ and Mg X $625.0 \AA$, contain only modes with periods longer than $10 \mathrm{~min}$. A detailed analysis of O v $629 \AA$ reveals that $3-4$ min oscillations that are above the $95 \%$ confidence level can be seen in many regions other than the sunspot umbra/plume.

\section{Observations and data}

The observations discussed here were taken over the period of 12-17 February 2004 by NIS, one of the components of CDS, on board SoHO (Harrison et al. 1995). Our observations used six spectral windows containing the following spectral lines: Si XII $520.8 \AA$, He I $522.2 \AA$, Fe XVI $335.4 \AA$, Fe XVI $360.8 \AA$, Mg IX 368.1 $\AA$, Mg X $625.0 \AA$, and O V $629.8 \AA$. The target of the observations was active region AR0554. The region contains a strong brightening in O v $629 \AA$ above the sunspot umbra. The intensity of the brightening is more than five times greater than the intensity in the surrounding area. Hence, the brightening can be classified as a sunspot plume. We have selected data taken on Feb. 12th, 13th, and 15th for the analysis in this paper. The data of each day consist of four 95-min time series and two raster images taken before and after the time-series observation. During each of the 95-min time series, the pointing of the detector was fixed in space while the Sun rotated under the field of view (FOV).
The exposure time is $30 \mathrm{~s}$, and the cadence is approximately $37 \mathrm{~s}$. The time-series observation of each day began at the following times: 07:03:02 (Feb. 12th), 07:59:48 (Feb. 13th), and 07:37:29 (Feb. 15th). Although NIS data provide good spectral resolution, the spatial resolution of NIS images is around $8^{\prime \prime}$. To better resolve fine features, we incorporated images from TRACE white light, $171 \AA$ and $1600 \AA$ passbands. In addition, we used MDI (Michelson Doppler Imager) magnetograms to obtain information on the photospheric magnetic fields.

TRACE images show that the observations cover a range of different features in the active region while MDI magnetograms indicate that the magnetic configurations in this region are highly dynamic. For instance, in Fig. 1, TRACE $171 \AA$ images show that there are distinctive multi-loop system below the sunspot on 15 Feb. However, the same region appears to be fuzzy on 13 Feb. From MDI magnetograms, it is noticeable that the concentrated positive magnetic fields on 13 Feb. become smaller and more spread out around the sunspot by 15 Feb.

The variation and diversity of the structures in the FOV provide an opportunity to compare oscillation properties in different features. Therefore, in addition to a examination of the whole region, we also selectively examined several individual pixels that reside in the following structures: a fanning-out loop system, the sunspot, a closed-loop system and a broad, fanlike open loop (see Sect. 4.2 for details). The selected locations are marked in Fig. 1 as long, rectangular boxes. Each of the boxes is divided into four sections, representing the FOV of the 
four time-series observations. The figure also shows discernible variations in the $\mathrm{O} v$ images, e.g., the plume becomes brighter and the number of bright regions increases over the four days.

\section{Analysis procedure}

\subsection{Calibration procedures}

NIS data were calibrated by the most up-to-date standard calibration routines in SolarSoft ${ }^{1}$, with the offset between NIS1 and NIS2 corrected using the routine nis_rotate. The lines in each spectral window are fitted by the following steps: we first averaged the data over time to create an averaged spectrum, then fitted the spectrum to obtain a set of fitting parameters, and lastly used these parameters as starting values for all data in the window.

As explained in CDS Software Note No. $53^{2}$, NIS line profiles were broadened after the recovery in October 1998. Therefore, each spectral line was fitted by a broadened Gaussian line profile function, and the line intensity was computed accordingly. The errors in the line intensities were computed based on the equations in CDS Software Note No. 49. For the time series, the trend of intensity variation was computed by a 25-point running average. The oscillations were extracted by subtracting the trend from the intensity variation (de-trend). The oscillation amplitudes depend not only on the driving source of the oscillations but also on the intensity of a spectral line. Since our interest is to investigate the driving source, we divided the oscillations by the trend to eliminate the dependency on the line intensity. The result of this is to produce what we call relative oscillations.

We selected TRACE and MDI images that have the observation times closest to the observation times of the NIS time series; hence, the features in the images are the best visual representation of our data. If TRACE $171 \AA, 1600 \AA$, and whitelight images (or MDI magnetogram and continuum images) were not taken at the same time, we calculated and corrected the difference in the coordinates based on the solar rotation rate at the location of the sunspot. The noise and cosmic ray contamination in TRACE images were reduced by a number of despike, de-streak, and smoothing routines. Since our observation was taken when SoHO instruments were turned $180^{\circ}$, MDI and NIS raster images were rotated to correct for this effect.

We then determined the coordinate offsets between the shifted NIS raster images and TRACE images by applying a cross-correlation method to align TRACE $171 \AA$ A passband images with the shifted NIS Mg IX $368 \AA$ raster. The TRACE white-light and $1600 \AA$ channel images were then shifted by the determined coordinate offsets. To align MDI images, we applied the cross-correlation method to align the MDI continuum with the shifted TRACE white-light images, and then shifted the magnetograms by the same amount.

\footnotetext{
${ }^{1}$ http://www.lmsal.com/solarsoft/

${ }^{2}$ http://solar.bnsc.rl.ac.uk/
}
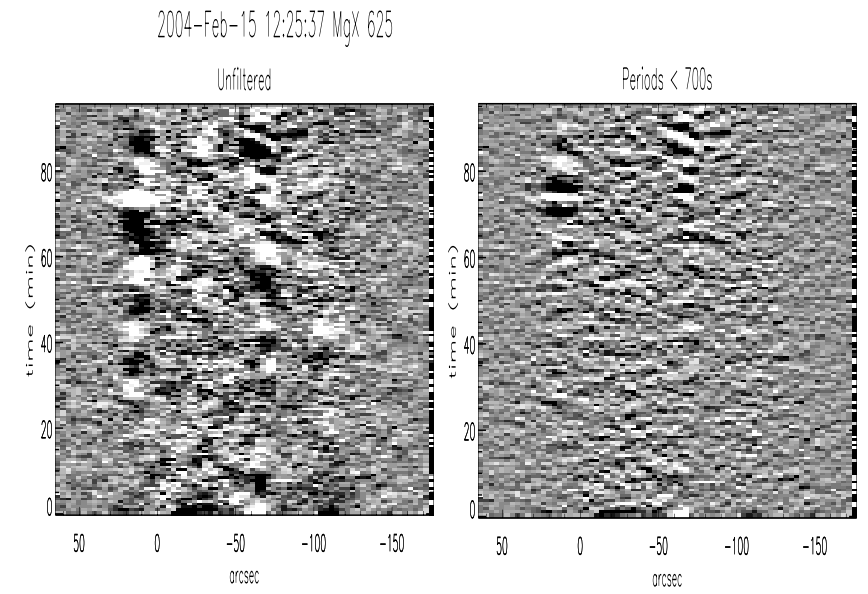

Fig. 2. The left panel is the de-trended X-T slice of Mg X $625 \AA$, on 15 February 2004 in AR0554. The right panel shows the result after oscillations with periods longer than $700 \mathrm{~s}$ are filtered out. The $X$-axis is the Solar $Y$ coordinates of the pixels along the slit, and the $Y$-axis is the time.

\subsection{Frequency analysis and filtering}

A wavelet transform is analogous to a localized Fourier transform. It provides information on the temporal dependence of a signal. Because the coronal oscillations often vary over time, the wavelet transform, which allows analysis of localized variations, is more suitable than the traditional Fourier analysis for our study. Details on the wavelet analysis may be found in Torrence \& Compo (1998) and O'Shea et al. (2001). Note that we used the standard Morlet wavelet as described by the above authors. To determine whether or not the oscillations found in the analysis are real, we implemented a randomization method (Linnell Nemec \& Nemec 1985) to estimate the significance level of the peaks in the wavelet spectrum. The significance level resulting from the randomization method has been compared with the significance level output from the procedure wavelet available from Torrence \& $\mathrm{Compo}^{3}$. The comparison shows that the former method is more strict than the latter. Nevertheless, the two results are consistent.

When a signal is composed of multiple oscillations with very different intensities, the weaker ones will be obscured by the stronger ones. Therefore, if we are interested in examining the weaker oscillations, the stronger ones need to be filtered out first. In this paper, we filtered out periods longer than $300 \mathrm{~s}$ to reveal weak 3-4 min oscillations. The filtered signals are called -filtered signals.

\section{Results}

\subsection{Examination of the entire FOV}

In our data, significant oscillations are detected in four lines, Si XII $520 \AA$, Mg X $625 \AA$, O v $629 \AA$, and He I $522 \AA$ with the dominant oscillations being for periods of $\approx 10 \mathrm{~min}$ or longer. While weaker oscillations with periods shorter than 10 min are commonly found in O v $629 \AA$, none of the other three lines

\footnotetext{
${ }^{3}$ http://paos.colorado.edu/research/wavelets/
} 

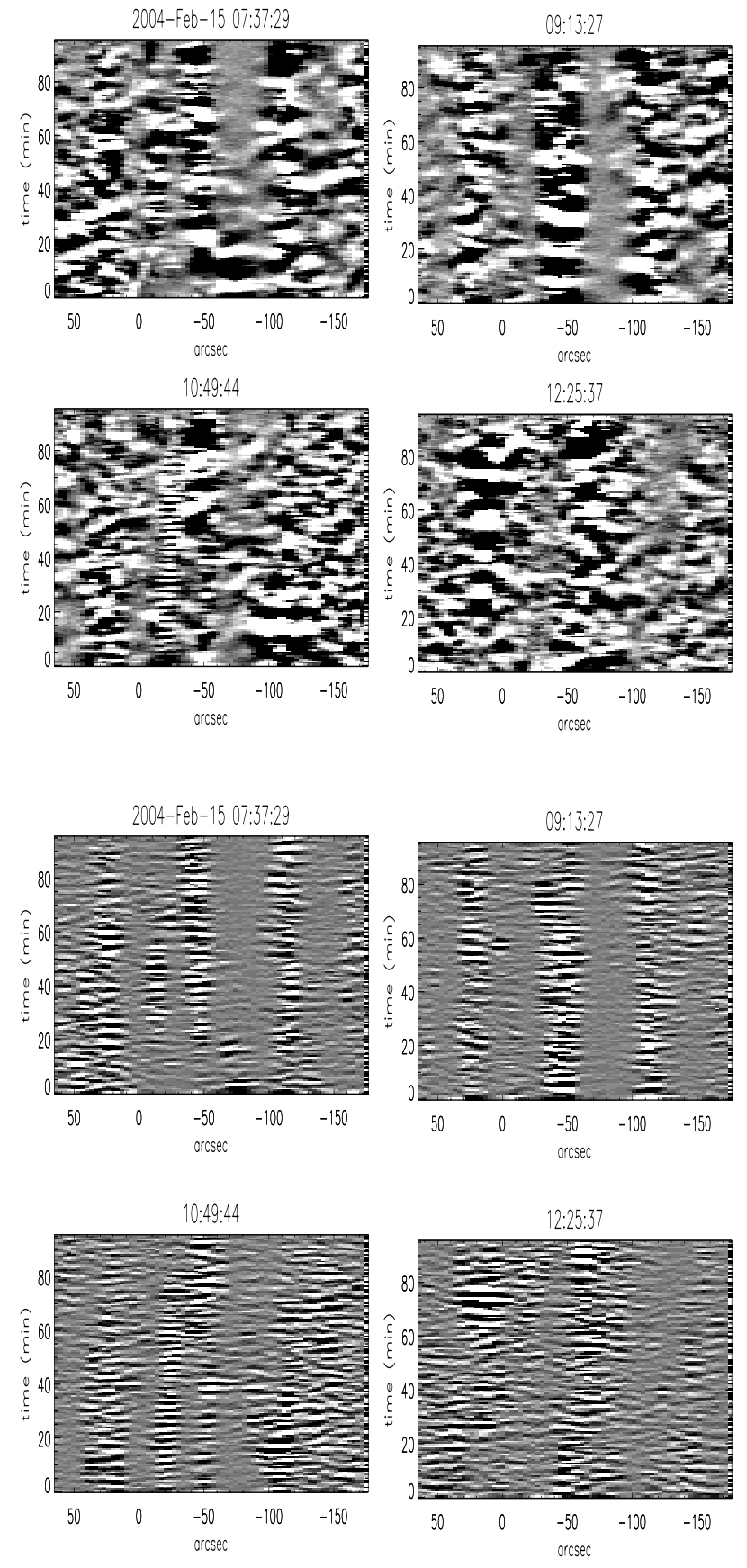

Fig. 3. The time series of unfiltered (top two rows) and $P 300$-filtered (lower two rows) oscillations of O v $629 \AA$. The starting time is indicated above each panel. The $X$-axis is the position along the slit.

exhibits oscillations with periods shorter than $600 \mathrm{~s}$. This distinction is demonstrated in Figs. 2 and 3.

In Fig. 2, we chose Mg X $625 \AA$ to show the case of longperiod oscillations. The left panel is the de-trended intensity variation over time along the slit (X-T slice). The $X$-axis is the Solar $Y$ coordinates of the pixels along the slit and the $Y$-axis is the time. The periodic brightenings and darkenings along the $Y$ axis indicate the existence of oscillations. We can see from this X-T slice that the period of the dominant oscillations is approximately $10 \mathrm{~min}$. After filtering out the oscillations with periods longer than $700 \mathrm{~s}$, we found that the data contains mainly noise (cf. the right panel). Interestingly, there is a small exception for the last $30 \mathrm{~min}$ of the observing sequence (at Solar $Y \sim 20^{\prime \prime}$ and $\sim-60^{\prime \prime}$ ), which corresponds to the edges of the sunspot (see Fig. 1). The absence of shorter-period oscillations could be an indication that the coronal structure in this region does not support them or that the intensities of these oscillations are below our detection limit.

For the short-period oscillations found in O v $629 \AA$, we selected the results of $15 \mathrm{Feb}$. to demonstrate the common properties of oscillations found in all three days. The X-T slices of O v $629 \AA$ are plotted in Fig. 3. The upper two rows are the unfiltered $\mathrm{X}-\mathrm{T}$ slices from the four observation times, and the images in the lower two rows are $P 300$-filtered X-T slices (i.e. oscillations with periods longer than $300 \mathrm{~s}$ are filtered out). The observation times are indicated above each panel. In the unfiltered images, the only location where one can see 3-min oscillations is in the panel of 10:49:44 UT at approximately $-20^{\prime \prime}$ to $-30^{\prime \prime}$, which is the location of a plume (cf. Fig. 1). However, small, short-period oscillations can often be seen on top of the dominant, long-period ones. The lower two rows in Fig. 3 (i.e. P300-filtered X-T slices) exhibit clear stripes of oscillations in many locations. Our wavelet analysis confirmed that most of these oscillations are above the $95 \%$ significance level and are of periods $\approx 238 \pm 30 \mathrm{~s}$. As a representative example, we show in Fig. 4 the wavelet analysis results of two locations, $-20.3^{\prime \prime}$ (which corresponds to the sunspot umbra) and $-111.0^{\prime \prime}$ (corresponding to a fan-like loop structure away from the sunspot), at the times of 10:49:44 UT and 07:37:29 UT, respectively, on 15 Feb. In each set of the upper two plots, the top panel is the de-trended signal along with errors; the middle panel is the corresponding wavelet spectrum; the lower panel is the probability level of the primary maxima in the wavelet spectrum; and the right panel shows the global wavelet spectrum, which is the sum of the wavelet power over time at each oscillation period. In the wavelet spectrum, the darker the color the higher the wavelet power, and the white dots mark the period of the primary maxima at each temporal point. In the lower two plots, the panels are the same as those stated above, except for the top panel, which shows $P 300$-filtered, relative oscillations (cf. Sects. 3.1 and 3.2 for the definition of relative oscillations and the filtering procedure). The signal inside the umbra (i.e. $-20.3^{\prime \prime}$ ) exhibits prominent 3-min oscillations with little or no noise with a life time $\sim 50 \mathrm{~min}$. In contrast, the signal at $-111.0^{\prime \prime}$, which is at a loop unrelated to the sunspot (cf. Fig. 1), has a dominant mode of $868 \mathrm{~s}$ before being filtered. Although some oscillations with smaller amplitudes and shorter periods can be seen on top of the dominant mode in the signal, they are much too weak, compared to the dominant one to be considered as significant. After filtering, we can see that these previously obscured minor oscillations have a period of 236 s with $98 \%$ significance level. Note that the wavelet power of these minor modes is comparable to the power of the 3-min oscillations at $-20.3^{\prime \prime}$ but with a much shorter life time $\approx 10-20 \mathrm{~min}$.

To examine the features underlying these oscillations, we compared TRACE and MDI images with NIS data. In Fig. 5, we show the TRACE $171 \AA$ images (top row), MDI magnetograms (second row), and NIS O V $629 \AA$ images (third row), along with the P300-filtered X-T slices (fourth row), all 

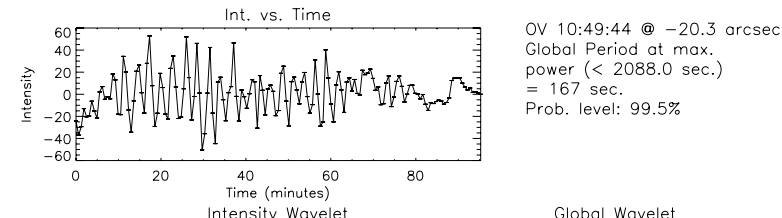
Global Period at max. power (< $2088.0 \mathrm{sec}$. $=167$ sec. $99.5 \%$
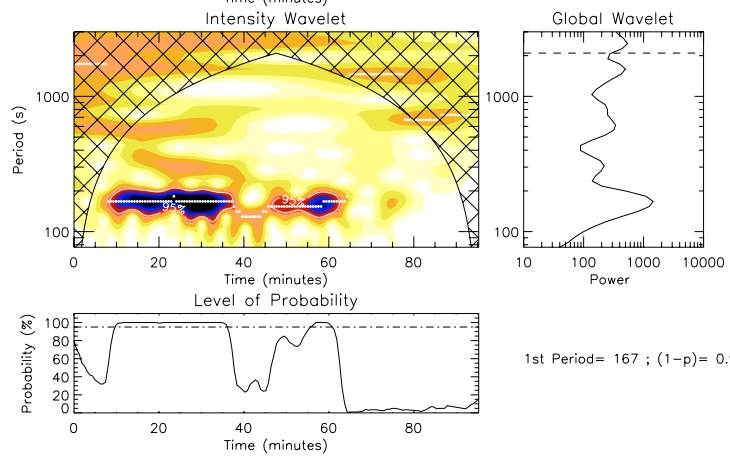

1st Period $=167 ;(1-p)=0.995000$

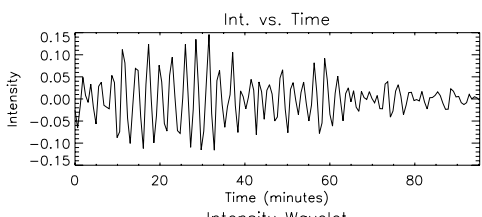

Rel. Osc. P<300s @ -20.3 arcsec Global Period at max. power $(<2088.0 \mathrm{sec}$. Prob. level: $99-100 \%$
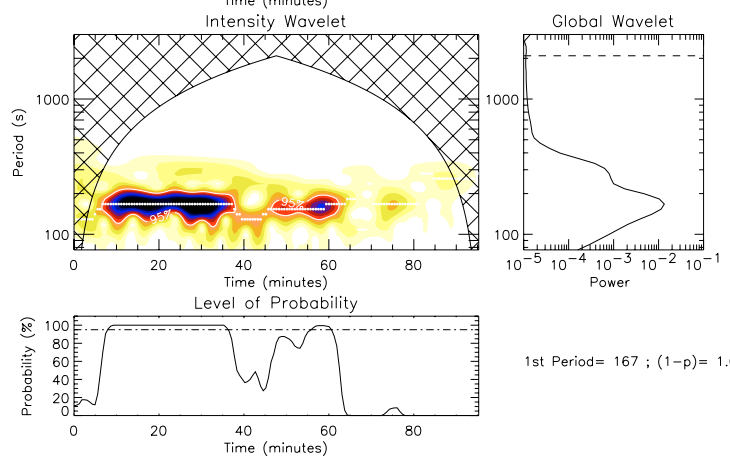

1st Period $=167 ;(1-p)=1.00000$

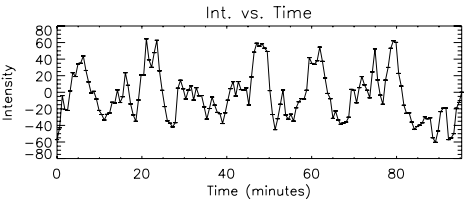

OV 07:37:29@-1111 arcsec Global Period at max.
power $(<2089.6$ sec $)$ $=868 \mathrm{sec}$. Prob. level: $99-100 \%$
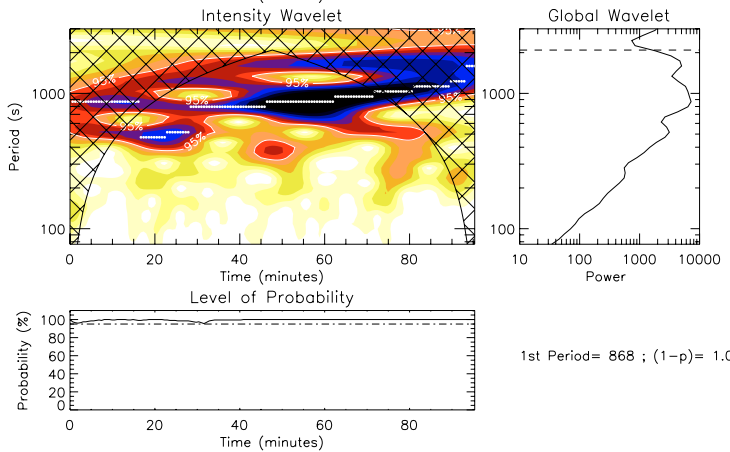

st Period $=868 ;(1-p)=1.00000$

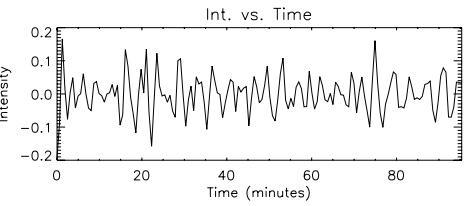

Rel. Osc. P<300s @ -111 arcsec Global Period at max. power $(<2089.6 \mathrm{sec}$. Prob. level: $98.5 \%$
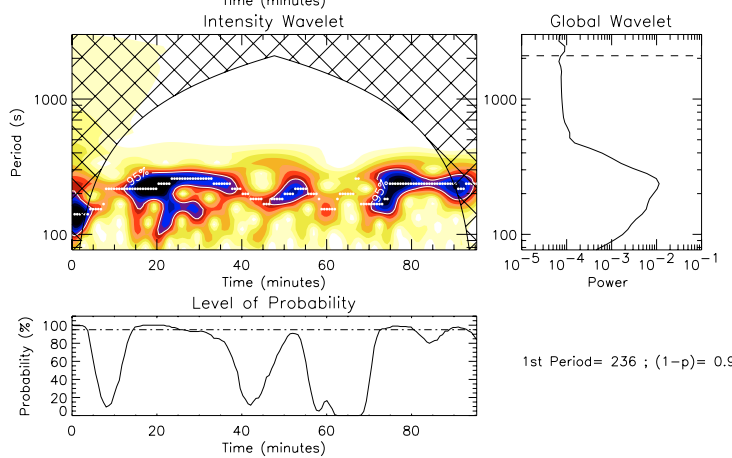

Fig. 4. The wavelet analysis on the locations inside (at $-20.3^{\prime \prime}$ ) and outside (at $-111.0^{\prime \prime}$ ) of the sunspot in O v $629 \AA$, as indicated in the upper right corner of each set of plots. In each of the upper two plots, the top panel shows the de-trended signals along with error bars, the middle panel is the corresponding wavelet spectrum, the bottom panel is the probability levels of the primary wavelet power at each temporal point, and the right panel is the global wavelet spectrum. The panels in the lower two plots are the same as above except for the top panel, which shows $P 300$-filtered, relative oscillations.

from 15 Feb. The lowest panels show the measured periods of the oscillations seen in the fourth row. The plus symbols correspond to the primary maxima in the global wavelet spectra at each pixel that are above the $95 \%$ significance level. We can see that there exist oscillations with periods $<250$ s at many locations outside of the umbra, such as magnetic-field concentrated regions, brightenings in O v $629 \AA$, and loops and footpoints. For instance, in the 1st column in Fig. 5, the location around $Y=-111^{\prime \prime}$, which is not connected with the sunspot but has some concentration of magnetic field (as revealed by MDI) and a brightening (as seen in the NIS map), exhibits the clear presence of 3-4 min oscillations. This suggests that 3-min oscillations are not a unique property of a sunspot plume/umbra, but may in fact exist in different locations within an active region, with some connection to the magnetic field. In Fig. 6, we compare the MDI magnetogram with the images of NIS O v $629 \AA$ and TRACE $1600 \AA$ band, which represent the features at the transition region and photosphere, respectively. We can see from the figure that the bright regions in both $1600 \AA$ and O v $629 \AA$ are correlated with the locations of strong magnetic fields. Specifically, the bright cell boundaries seen in the $1600 \AA$ image map very well with the magnetic network lines visible in the magnetogram (e.g. at around $20^{\prime \prime}$ between the two white horizontal lines). Comparing these images with the stripes of 3-min oscillations, we can see that the stripes are often located at these bright cell boundaries, which are also the bright regions in $\mathrm{O} v$. The bright dots around the sunspot, as seen in the magnetogram, are likely to be the moving magnetic features (MMFs), which are small magnetic elements moving out continuously from sunspots and appearing in both polarities (Lee 1992). Thus, the two stripes of 3-min oscillations located between $0^{\prime \prime}$ and $-50^{\prime \prime}$, which coincide with the bright dots, may be related to MMFs. This figure further confirms that these oscillations are related to magnetic structures. The reason that 3-min oscillations have not been widely found in the regions not directly linked with a sunspot umbra could be that 3-min oscillations are often weaker than longer-period ones and therefore difficult to detect. A sunspot plume/umbra 

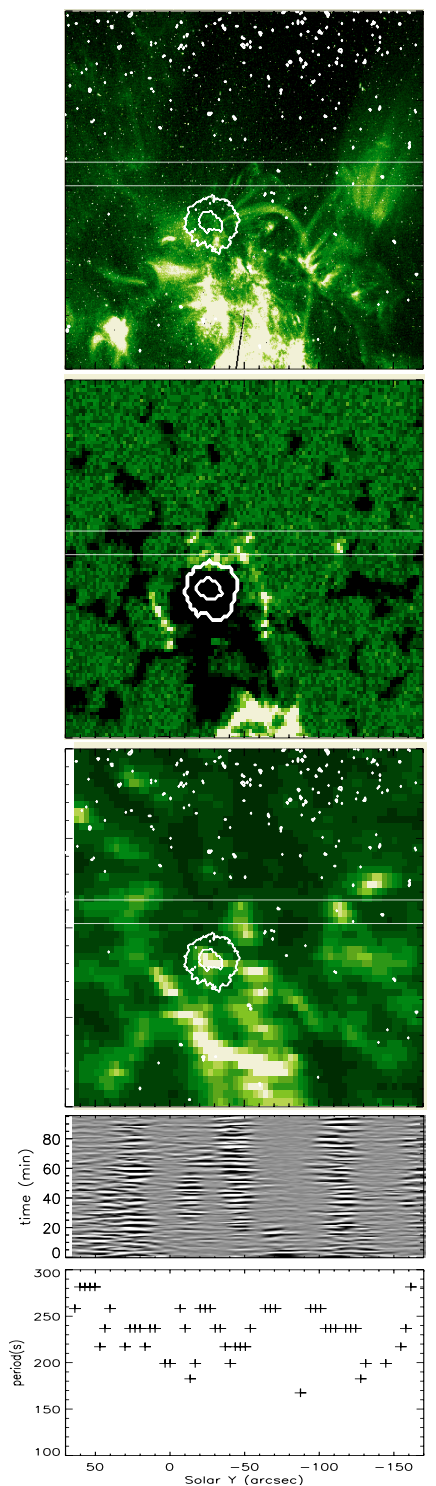
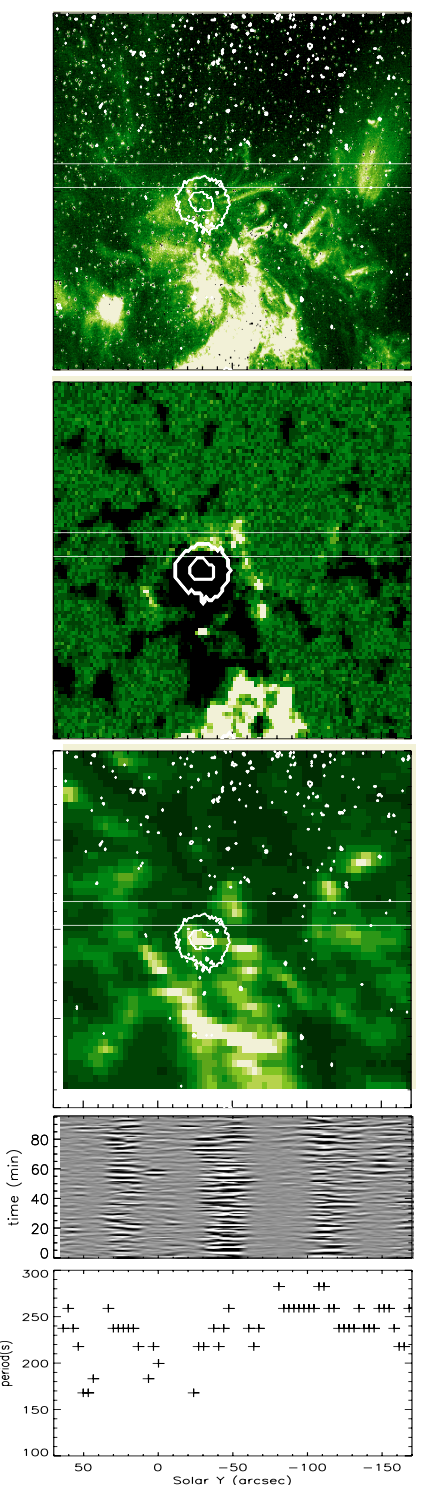
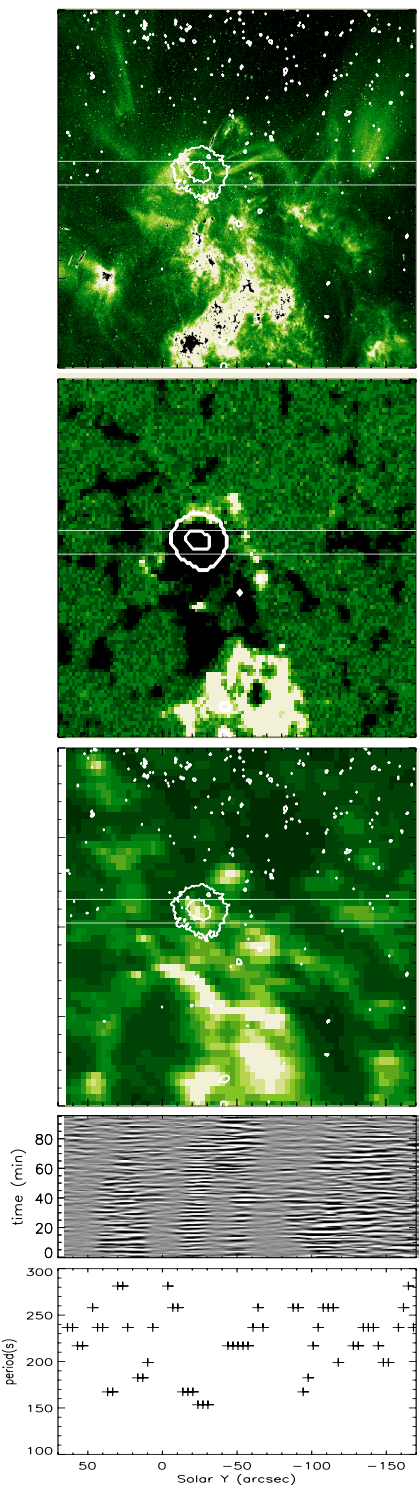
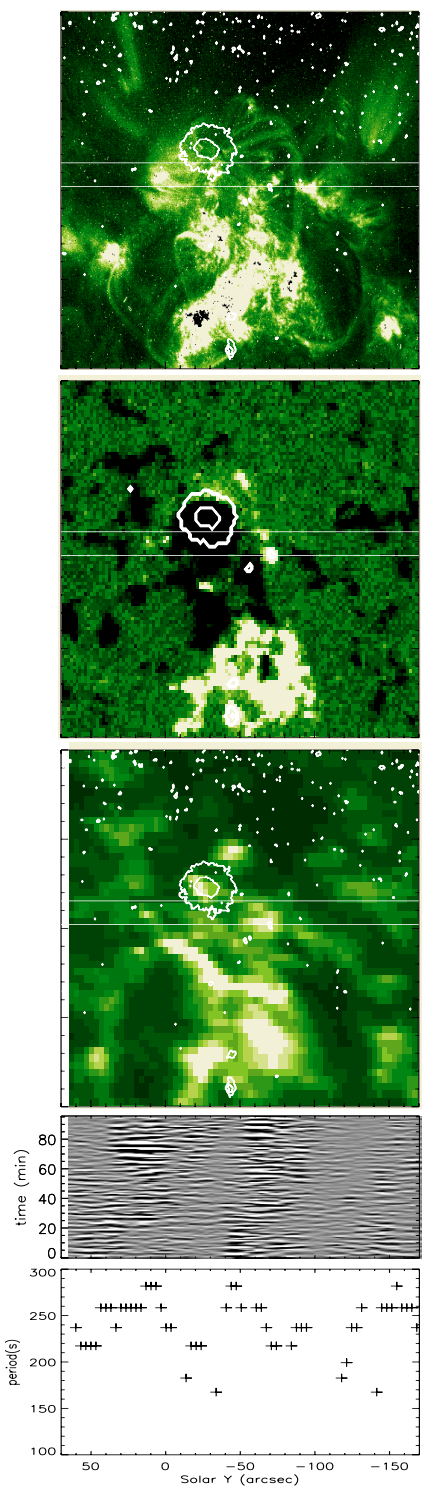

Fig. 5. The images and filtered oscillations from $15 \mathrm{Feb}$. Note that the images are rotated $90^{\circ}$ anti-clockwise compared to Fig. 1 for the convenience of comparing features with the locations of oscillations. Therefore, the vertical axis corresponds to Solar $X$, covering a FOV of 200", and the horizontal axis is Solar $Y$. The values of the Solar $Y$ coordinates are indicated in the bottom row. From the top row down, the images are: TRACE $171 \AA$, MDI magnetogram, NIS O v $629 \AA$ raster image, X-T slices of $P 300$-filtered oscillations, and the primary oscillation periods of the X-T slices. The contours in the images are the outlines of the umbra and penumbra, and the two horizontal lines mark the beginning and ending locations of the slit in each time-series observation. The four columns (from left to right) correspond to the following observation times: 07:37:29, 09:13:27, 10:49:44, and 12:25:37 for 15 Feb. 2004.

may act as a frequency filter and enhancer that magnifies 3-min oscillations and filters out all longer-period oscillations.

\subsection{Examination of individual pixels}

We have selected the following four regions for a detailed examination: a fanning-out loop system with one footpoint in the sunspot (Region 1: Solar $Y>0^{\prime \prime}$ ); the sunspot region (Region 2: Solar $Y \approx-20^{\prime \prime}$ to $-30^{\prime \prime}$ ); a dynamic, closed-loop system with one footpoint changing location over time and the other one rooted in the sunspot (Region 3: Solar $Y \approx-48^{\prime \prime}$ to $\left.-90^{\prime \prime}\right)$; and a broad, fuzzy, fan-like loop structure that is not directly linked to the sunspot (Region 4: Solar $Y \approx-111^{\prime \prime}$ to $\left.-134^{\prime \prime}\right)$.

Significant 10-15 min oscillations are the dominant oscillations in all unfiltered signals, except for the signals in the umbra, which show 3-min oscillations as the dominant one. Most of the "3-min" oscillations outside of the umbra have periods between $200 \mathrm{~s}$ and $240 \mathrm{~s}$. A close examination of the features and oscillations at these selected regions reveals that the 3-4 min oscillations often appear in the features that are above or near strong magnetic fields. Intriguingly, we did not find significant 3-4 min oscillations in the penumbra, which shares the same magnetic field region with the umbra. Most of the oscillations in the penumbra are either below the $95 \%$ 

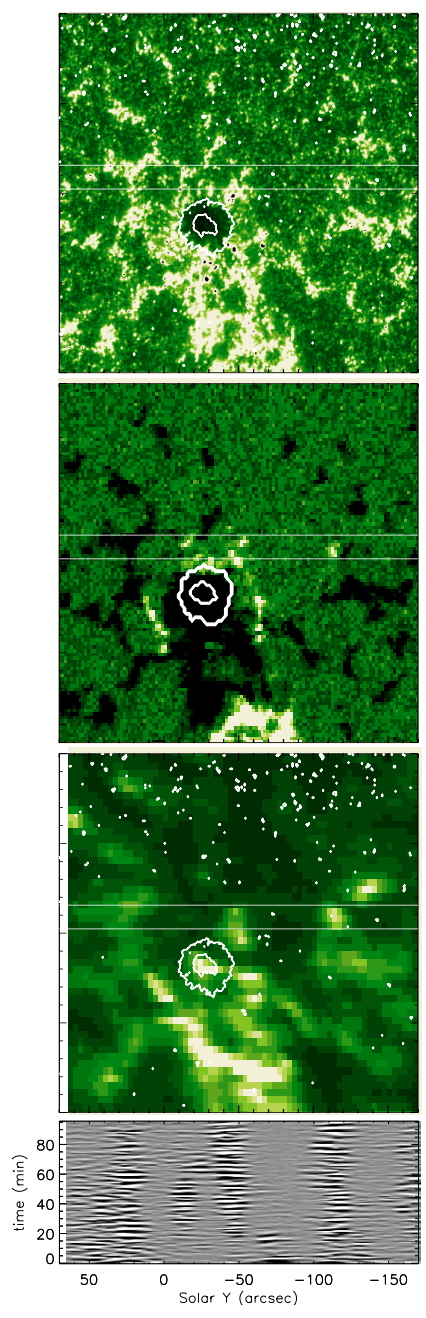

Fig. 6. A comparison of the TRACE $1600 \AA$ channel image (top panel), MDI magnetogram (second), NIS O v $629 \AA$ image (third), and the P300-filtered $\mathrm{X}$-T slice (bottom). The contours and the horizontal lines are as described in Fig. 5. The observation time is 07:37:29. In the magnetogram, the white dots around the sunspot probably correspond to the moving magnetic features. The supergranulation cell boundaries are visible in the TRACE image as bright regions. The locations of these boundaries match the locations of the 3-min oscillation stripes.

significance level or of periods longer than $250 \mathrm{~s}$. One possible reason for the absence of the oscillations in the penumbra is that the region is dark in O V $629 \AA$; hence, the oscillations are too weak to be detected. This leads to a speculation that the 3-4 min oscillations might exist in all features but are only observable in the regions of strong emission. The significant "3-min" oscillations outside of the umbra often last for approximately 15-30 min while the oscillations in the umbra are stable and remain above the $95 \%$ significance level for longer than $50 \mathrm{~min}$. One possible explanation is that the duration of the oscillations reflects the spatial size of the features being detected. Since our detector did not follow the solar rotation, the oscillation source regions quickly move out of our FOV if their spatial scales are of few arcsec. As can be seen in Figs. 1 and 5, the magnetic fields, brightenings, and loops are often smaller than $10^{\prime \prime}$, and correspondingly in those locations the life time of the oscillations are $\approx 10-20$ mins.

Lastly, we would like to point out that the significance level of dominant oscillations is based on the global wavelet power of the oscillations. Hence, if the oscillations are only significant for a short period of time, the significance level of the global wavelet of these oscillations may be below the $95 \%$ significance level.

\section{Discussion and conclusions}

In recent years, with the advent of high resolution Extreme UltraViolet (EUV) imagers and spectrographs, there has been a increase in the number of observations of the oscillations in the transition region and the corona above active regions. Acoustic waves propagating in coronal loops were first noticed in Extreme ultraviolet Imaging Telescope (EIT) observations (Berghmans \& Clette 1999). A number of propagating waves were also analysed by De Moortel et al. (2002) from TRACE data, starting in active regions and propagating upwards into diverging, fan-like bundles of loops that fade out with height (similar to our region 1). Multi-wavelength observations with EIT and TRACE confirmed that the diverging fan structures consist of multiple loop threads with different temperatures and corresponding sound speeds (Robbrecht et al. 2001; King et al. 2003). Time periods of $P=172 \pm 32 \mathrm{~s}$ were reported for loops rooted near sunspots (our region 2 and 3), which coincide with the 3-min p-mode oscillations detected in sunspots (Brynildsen et al. 2002, 2000; Fludra 2001; Maltby et al. 2001; Banerjee et al. 2002), while waves that start further away from sunspots (active region plages, our region 4) have periods of $P=321 \pm 74 \mathrm{~s}$, which coincide with the global 5-min p-mode oscillations. O'Shea et al. (2002) found the presence of oscillations in a wide range of temperatures (from the temperature minimum of the solar atmosphere to the temperature of the upper corona) and time delays between the low- and hightemperature oscillations. These results clearly indicate that subphotospheric $\mathrm{p}$-mode oscillations penetrate through the chromosphere and transition region, and excite coronal acoustic type waves. De Pontieu et al. (2003) have reported strong intensity oscillations in the upper transition region footpoints of hot coronal loops. They report a range of periods from 200 to $600 \mathrm{~s}$, typically lasting for 4-7 cycles. The oscillations occur at the periphery of the plage regions (our region 4), which the authors associate with moss. They further indicate a good correlation with the $\mathrm{p}$-modes in the photosphere and $\mathrm{H} \alpha$ flows in spicules. Some of these studies give an indication of the origin of these oscillations.

In this work, we examined the active region, AR0554, which contains various types of loop systems and a sunspot plume. The images of MDI magnetograms, NIS O V $629 \AA$, and TRACE $171 \AA$ show that the structures of the active region (from the photospheric level to corona) changed over the time of our observation. Among all the NIS spectral lines analysed, significant oscillations were only found in the following lines: Si XII $520 \AA$, Mg X $625 \AA$, O v $629 \AA$, and He I $522 \AA$. The periods of the strongest oscillations in these lines are

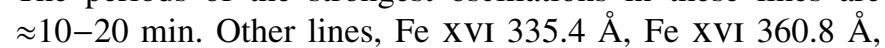
and Mg IX 368.1 $\AA$, although formed at similar temperatures to Si XII $520 \AA$ and $\mathrm{Mg}$ x $625 \AA$, do not exhibit any significant oscillations. After filtering out the dominant 10-20 min oscillations from the four lines, Si XII $520 \AA$, Mg x $625 \AA$, O V $629 \AA$, and He I $522 \AA$, we found that there are significant (i.e. above $95 \%$ significance level) shorter-period oscillations in O v $629 \AA$ but not in the other three lines. Specifically, we found that weak but significant 3-4 min oscillations are not confined to the umbra/plume but can be seen in many bright 
locations in O v $629 \AA$. Although we do not have enough spatial resolution in CDS and are limited by the detection limit of the full disk magnetogram, the close association of brightenings seen in the NIS O V raster and magnetic field concentration at different regions (see Sect. 4.2.1) do indicate a possible influence of the magnetic field on the existence of these observed oscillations. From TRACE $1600 \AA$ channel images, we found close correspondence between the locations of the oscillations and the supergranulation cell boundaries, which coincide with the magnetic network lines. This further indicates that these oscillations are related to magnetic structures.

In a recent simulation De Pontieu et al. (2005) have shown that photospheric oscillations with a acoustic cut-off frequency around $4.8 \mathrm{mHz}$ or slightly lower can actually tunnel through to higher up in the atmosphere so long as they are guided along an inclined magnetic flux tube. This non-verticality of the flux tube decreases the cut-off frequency, allowing the lower frequency modes to tunnel through. Apart from the sunspot in our active region we come across different kind of inclined magnetic flux tubes (loops) which can certainly act as wave guides.

We should also point out that these 3-4 min oscillations outside of the umbra do not last very long. Their significance level often drops below $95 \%$ in less than $30 \mathrm{~min}$. Therefore, they appear in a signal as intermittent oscillations with durations of $\approx 15-30 \mathrm{~min}$. Since rotational compensation was not applied, if an oscillating region has a coherence length of few arcsec, the region would enter and exit our 4 " wide slit in $40 \mathrm{~min}$ or so. In contrast, the 3 -min oscillations at the umbra are strong and stable for longer than $50 \mathrm{~min}$ (in this case the oscillating region is much wider). The non-detection of 3-min oscillations in coronal CDS lines by Brynildsen et al. (2002) yet being observable in TRACE 171 could be due to the relatively coarse resolution of CDS as compared to the size of the oscillating region at coronal heights. An alternative explanation could be the formation process of the Fe IX $171 \AA$ in the TRACE filter. A non-Maxwellian electron distribution makes a significant fraction of the plasma in the TRACE $171 \AA$ passband to be derived from temperatures around $\approx 300000 \mathrm{~K}$, as opposed to $\approx 800000 \mathrm{~K}$ (Doyle et al. 2004).

To find the possible sources of the 3-4 min oscillations outside of the umbra, we compared the oscillations of single pixels in different regions. We suggest that 3-min oscillations can exist in many magnetic structures. However, they are often too weak to be seen in an unfiltered signal. Only in sunspot umbrae/plumes, do they become prominent. The reason could be that the structure of an umbra/plume enhances 3-min oscillations while suppressing oscillations at other longer periods.

Acknowledgements. We would like to thank CDS, MDI, and TRACE teams at Goddard Space Flight Center for their help in obtaining the present data. CDS and MDI are part of SoHO, a mission of international cooperation between ESA and NASA. Research at Armagh Observatory is grant-aided by the N. Ireland Dept. of Culture, Arts and Leisure. This work was supported in part by a PRTLI research grant for Grid-enabled Computational Physics of Natural Phenomena (Cosmogrid). We wish to thank the Royal Society London and the British Council for funding of visits between the UK and India. D.B. is also supported by the Indo-French project (No. 2504-3). We also thank the referee for a range of comments which has greatly improved the paper.

\section{References}

Banerjee, D., O'Shea, E., Goossens, M., Doyle, J. G., \& Poedts, S. 2002, A\&A, 395, 263

Berghmans, D., \& Clette, F. 1999, Sol. Phys., 186, 207

Brynildsen, N., Maltby, P., Fredvik, T., \& Kjeldseth-Moe, O. 2002, Sol. Phys., 207, 259

Brynildsen, N., Maltby, P., Leifsen, T., Kjeldseth-Moe, O., \& Wilhelm, K. 2000, Sol. Phys., 191, 129

De Moortel, I., Ireland, J., Hood, A. W., \& Walsh, R. W. 2002, A\&A, 387, L13

De Pontieu, B., Erdélyi, R., \& De Moortel, I. 2005, ApJ, 624, L61

De Pontieu, B., Erdélyi, R., \& de Wijn, A. G. 2003, ApJ, 595, L63

Doyle, J. G., Madjarska, M. S., Dzif̌ćáková, E., \& Dammasch, I. E. 2004, Sol. Phys., 221, 51

Fludra, A. 2001, A\&A, 368, 639

Harrison, R. A., Sawyer, E. C., Carter, M. K., et al. 1995, Sol. Phys., 162,233

King, D. B., Nakariakov, V. M., Deluca, E. E., Golub, L., \& McClements, K. G. 2003, A\&A, 404, L1

Lee, J. W. 1992, Sol. Phys., 139, 267

Linnell Nemec, A. F., \& Nemec, J. M. 1985, AJ, 90, 2317

Maltby, P., Brynildsen, N., Fredvik, T., Kjeldseth-Moe, O., \& Wilhelm, K. 1999, Sol. Phys., 190, 437

Maltby, P., Brynildsen, N., Kjeldseth-Moe, O., \& Wilhelm, K. 2001, A\&A, 373, L1

O'Shea, E., Banerjee, D., Doyle, J. G., Fleck, B., \& Murtagh, F. 2001, A\&A, 368, 1095

O’Shea, E., Muglach, K., \& Fleck, B. 2002, A\&A, 387, 642

Robbrecht, E., Verwichte, E., Berghmans, D., et al. 2001, A\&A, 370, 591

Torrence, C., \& Compo, G. P. 1998, Bull. Am. Meteorolog. Soc., 79, 61 